This is the final peer-reviewed accepted manuscript of:

Effects of ionic liquids on membrane fusion and lipid aggregationof egg-PC liposomes Paola Galletti, Danilo Malferrari, Chiara Samorì, Giorgio Sartor, Emilio Tagliavini, Colloids and Surfaces B: Biointerfaces 125 (2015) 142-150

The final published version is available online at: http://dx.doi.org/10.1016/j.colsurfb.2014.11.021

Rights / License:

The terms and conditions for the reuse of this version of the manuscript are specified in the publishing policy. For all terms of use and more information see the publisher's website.

This item was downloaded from IRIS Università di Bologna (https://cris.unibo.it/)

When citing, please refer to the published version. 


\title{
Effects of ionic liquids on membrane fusion and lipid aggregation of egg-PC liposomes
}

\author{
Paola Galletti ${ }^{\mathrm{a}, \mathrm{b}}$, Danilo Malferrari ${ }^{\mathrm{b}, *}$, Chiara Samorì ${ }^{\mathrm{b}}$, \\ Giorgio Sartor ${ }^{\mathrm{b}, \mathrm{c}}$, Emilio Tagliavini ${ }^{\mathrm{a}, \mathrm{b}}$ \\ a Dipartimento di Chimica “G. Ciamician” Alma Mater Studiorum, Università di Bologna, via Selmi 2, 40126 Bologna, Italy \\ ${ }^{\mathrm{b}}$ Centro Interdipartimentale di Ricerca Industriale Energia e Ambiente (CIRI), via S. Alberto 163, 48123 Ravenna, Italy

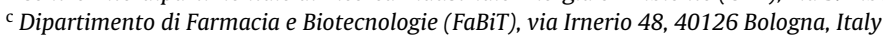

\section{Keywords:}

Ionic liquids

Egg-PC membrane fusion

Pyrene fluorescence

\begin{abstract}
A B S T R A C T
In this study we have explored the effects of different groups of ionic liquids (ILs) on membrane fusion. The ILs used contain different head groups: N-methylimidazolium, 3-methylpyridinium and $\mathrm{N}$-methylpyrrolidinium; short alkyl or ether functionalized side chains (with one or two ethoxy functionalities), paired with chloride anion. These ILs have been compared with 1-dodecyl-3-methylimidazolium bromide as example of a highly lipophilic IL. The effect of ILs on membrane fusion was investigated through pyrene steady state fluorescence probing, using the IE factor and excimer/monomer ratio (IE/IM) as parameters. The ratio between the vibronic bands of pyrene $\left(I_{1} / I_{3}\right.$ ratio) has been used to monitor the effect of ILs on the aggregation properties of egg-PC liposomes. The effect of different ILs' families was evident; the pyridinium ILs induced a greater extent of fusion than pyrrolidinium and imidazolium ILs having the same side chain. Marginal effect could be attributed to different anions. ILs with short alkyl chains were usually more effective than ether functionalized ones. The aggregation behaviors of ILs having dioxygenated chains have been measured in buffer solution.
\end{abstract}

\section{Introduction}

Ionic liquids (ILs) have received considerable interest because of their unique physico-chemical properties such as very low volatility, wide electrochemical window, non-flammability, high thermal stability and wide liquid temperature range [1,2]. ILs have been explored as alternative media for chemical reactions [3] and recently the applications on small industrial scale gained the first successful developments in pilot plants [4]. ILs have been claimed to be green solvents, despite their wide chemical composition and diversity before reaching a deeper knowledge of their behavior in the environment. In the last decade many studies have been conducted dealing with ILs properties such as ecotoxicity [5-7], biodegradability [8], biological effects on microorganisms [9], lipophilicity [10], physicochemical properties [11] and interaction with sediments [12,13]. Due to their amphiphilic nature, some ILs have been also used as solvents for self-assembling structures such as micelles [14,15], reverse micelles, as phase transfer

\footnotetext{
* Corresponding author.

E-mail address: danilo.malferrari2@unibo.it (D. Malferrari).
}

catalysts [16] and amphiphilic self-assembling media [17]. In the literature there is plenty of data about the measurements of the micellar properties of ILs with long alkyl chains [18-20], but little is known about the behavior of ILs with short-chains or with chains containing oxygen atoms. Moreover, poor attention has been paid to the effects that ILs have on cell membrane properties, either using living cells or membrane models. Studies on changes in ILs structures pointed out the attention on their effect on model membranes [21,22]. Thus a detailed knowledge of ILs' effects and mode of action toward membranes, that are the interface between the cell and the environment, is needed for a deep evaluation of ILs biological effects.

Initial investigations on membrane effects of ILs with different alkyl chain lengths [22] highlighted that imidazolium ILs with alkyl chains longer than six carbon atoms deeply alter a membrane bilayer, inducing leakage of content and completely disrupting the model membrane at concentrations near $100 \mathrm{mM}$. ILs with shorter alkyl chains (four carbon atoms), even at concentrations up to $500 \mathrm{mM}$, induce a slow and little leakage of content that is not indicative of membrane disruption. Similar results have been recently reached in another study employing model membranes as biomimetic system and the microorganism Shewanella oneidensis [23]. In two recent studies 
a small group of ILs, 1-octyl-3-methylimidazolium chloride, 1-buytl-3-methylimidazolium tetrafluoroborate and 1-benzyl-3methylimidazolium tetrafluoroborate have been evaluated on dipalmytoylphosphatidylcholine (DPPC) and dimyristoylphosphatidylcholine (DMPC) liposomes [24,25]. The authors found that a long chain IL (1-octyl-3-methylimidazolium chloride) reduces the phase transition temperature of lipids and high molar ratio causes fusion of solid gel and liquid crystalline phases of liposomes. A short chain IL does not appreciably influence the phase transition temperature in the studied molar ratio.

ILs with different cationic head group and chain length have been recently studied and exhibit distinct mechanisms of membrane interaction, insertion and disruption that could be correlated with their biological activities, depending on the lipid nature and membrane lipid domains [26]. The results indicate, in particular, that both the ILs side chain composition (number and presence of oxygen atoms) and the ILs head-groups are important elements for membrane activity and cell toxicity.

In this paper we investigate the effects that ILs with different cationic head groups, anions and side-chain length have on membrane fusion using pyrene (Py) as fluorescence probe, considering also the presence of ethoxy units in the side chain of the cation moiety. Membrane fusion is commonly defined as a process that transforms two lipid bilayers from topologically distinct compartments into one connected compartment [27].

Liposomes of L- $\alpha$-phosphatidylcholine (Egg-PC) were used as models for cell membranes [28]. The ILs used are composed by $\mathrm{N}$-methylimidazolium 1, 3-methylpyridinium $\mathbf{2}$ or $\mathrm{N}$-methylpyrrolidinium $\mathbf{3}$ head groups, functionalized with short alkyl chains (butyl a, ether side chains (2-methoxyethyl b, 2(2-methoxyethoxy)ethyl c)) and with chloride, tetrafluoroborate or dicyanamide as counter anions. The head group structures and one of the three ILs families are depicted in Chart 1. For comparison we have also tested an IL with a long alkyl chain, 1-dodecyl-3-methylimidazolium bromide (1f), whose aggregation properties are well known and described in the literature [29-31].

The ether-functionalized imidazolium-based ILs $\mathbf{1 b}$ and $\mathbf{1 c}$ are a promising class of alternative solvents with some interesting properties, such as high solubility for carbohydrates [32], suitable features as reaction media for some biocatalytic processes [33] and for catalytic asymmetric reactions [34], capability to be exploited in dye-sensitized photoelectrochemical solar cells [35] and nanoparticles stabilizing properties [36]. In previous papers we have demonstrated that the introduction of one oxygen atom into the lateral chain of imidazolium-based ILs decreases their toxicity with respect to the alkyl counterparts toward the crustacean Daphnia magna and the bacterium Vibrio fischeri [37] although it also decreases their biodegradability in soils [38]. Herein a further goal was to verify if the previously observed trend of reducing toxicity by increasing the polarity of the cation [21] can be related to membrane fusion.

\section{Experimental}

\subsection{Materials}

All reagents and solvents used were purchased from Aldrich; 1-methylimidazole, 3-methylpyridine, 1-methylpyrrolidine, 1chlorobutane and 2-chloroethyl methyl ether were re-distilled before use to avoid the formation of colored impurities in ILs (see Supplementary Material for purification details). Pyrene was purchased from Fluka, Milan, Italy. Milli-RO water (resistivity $18.2 \mathrm{M} \Omega \mathrm{cm}$ at $25^{\circ} \mathrm{C}$; filtered through a $0.22 \mu \mathrm{m}$ membrane) was used for sample preparation.
Synthetic and purification procedures of ILs are reported in the Supplementary Material. ILs used in the experiments were dried overnight at $80^{\circ} \mathrm{C}$ under vacuum just before the experiments. The stock solution of Py in ethanol $(3 \mathrm{mM})$ was prepared daily and stored in the dark at $4{ }^{\circ} \mathrm{C}$ for one night before usage. For the preparation of buffer solution containing Py, an aliquot of ethanol solution containing Py was used, the solvent was evaporated under vacuum and then an adequate volume of buffer was added to obtain the concentration of $3 \mu \mathrm{M}$ [39]. The different ILs concentrations were obtained by adding progressive amounts of ILs to the same volume of buffer solution containing Py and egg-PC liposomes. The ratio of Py: total phospholipids was $1: 100(\mathrm{~mol} / \mathrm{mol})$, and the total phospholipids concentration was $0.25 \mathrm{mM}$ in all cases.

\subsection{Liposome preparation and characterization}

The egg-PC liposomes preparation procedure was adapted from previously described procedures [28,39]. Egg-PC was dissolved in a chloroform/methanol mixture $(3: 1, \mathrm{v} / \mathrm{v})$ and dried under vacuum at room temperature for $4 \mathrm{~h}$ to obtain a thin film. The film was hydrated in $140 \mathrm{mM} \mathrm{NaCl}, 10 \mathrm{mM}$ Tris $\mathrm{HCl}, 0.1 \mathrm{mM}$ EDTA, $\mathrm{pH} 8.0$, vigorously vortexed for $7 \mathrm{~min}$, and subjected to 20 cycles of extrusion through $100 \mathrm{~nm}$ pore size polycarbonate membranes (nominal), with a Lipofast syringe-type extruder (Avestin, Ottawa, Canada) [39]. Egg-PC liposomes mean dimensions (133 nm) have been characterized by Dynamic Light Scattering (DLS) employing a Malvern Nano ZS instrument with a $633 \mathrm{~nm}$ laser diode; data are reported in the Supplementary Fig. S1.

\subsection{Steady state fluorescence measurements of membrane fusion and scattering}

Membrane fusion measurements using the IE factor were adapted from the method of Schoen et al. [39] and were carried out with a Jasco spectrofluorometer FP-6200 equipped with a thermostated cuvette holder and a magnetic stirring device. Two groups of egg-PC liposomes were used. An aliquot of egg-PC liposomes was loaded with Py and then combined with an aliquot of nude lipid vesicles. Final Py concentration in buffer solution was $3 \mu \mathrm{M}$. The emission spectra excited at $334 \mathrm{~nm}$ were recorded in the $350-550 \mathrm{~nm}$ range (right-angle geometry) and the fluorescence intensities of the monomer (IM) $(395 \mathrm{~nm}$ ) and excimer (IE) $(480 \mathrm{~nm})$ emission peaks were measured. Excitation and emission slits with a nominal bandpass of $5 \mathrm{~nm}$ were used for all measurements [40]. The ILs induced fusion of lipid vesicles was performed by adding nude egg-PC liposomes to Py-loaded egg-PC liposomes $(1: 2, w t / w t)$ in the presence of ILs at different concentrations $(30 \mu \mathrm{L}$ of egg-PC liposome solution). Fusion induced by ILs was expressed as percentage relative to the fusion induced by Triton $\mathrm{X}-100$, added at $0.01 \%(\mathrm{v} / \mathrm{v})$. Relative fluorescence was obtained by calculating $100 \times\left(\mathrm{IE}_{0}-\mathrm{IE}_{x}\right) /\left(\mathrm{IE}_{0}-\mathrm{IE}_{\mathrm{tot}}\right)$, where IE represents the excimer fluorescence intensity (IE) during fusion, and $\mathrm{IE}_{0}$ and $\mathrm{IE}_{\text {tot }}$ represent, respectively, the intensities at zero concentration and after the addition of Triton X-100. All the experiments were performed at $25^{\circ} \mathrm{C}$. All fluorescence spectra were background subtracted using an appropriate blank.

The excimer to monomer fluorescence intensity ratio (IE/IM) was determined by measuring fluorescence intensity at the monomer (IM) and excimer (IE) peaks. The first $\left(I_{1}\right)$ and third $\left(I_{3}\right)$ vibronic peaks of Py appear at $375 \mathrm{~nm}$ and $385 \mathrm{~nm}$, respectively. Py $\Delta I$ values in buffer solution and in egg-PC liposomes were calculated using the following equation:

$\Delta I=I-I_{0}$ 

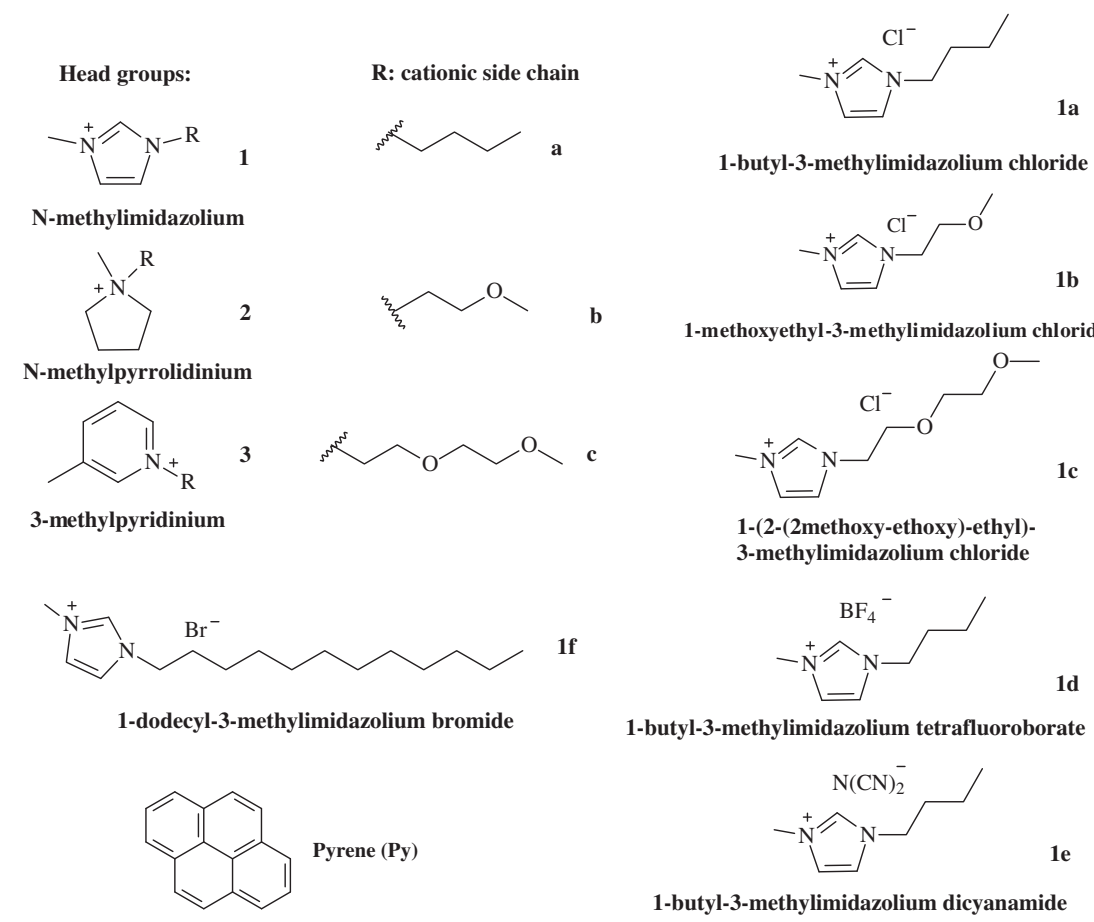

1-butyl-3-methylimidazolium chloride

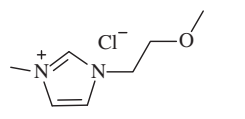

$1 \mathbf{b}$

1-methoxyethyl-3-methylimidazolium chloride

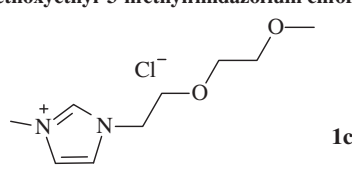

1-(2-(2methoxy-ethoxy)-ethyl)-

3-methylimidazolium chloride

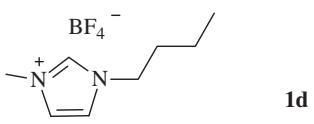

1-butyl-3-methylimidazolium tetrafluoroborate

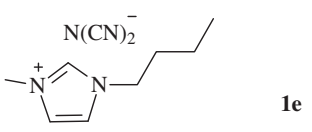

1-butyl-3-methylimidazolium dicyanamide

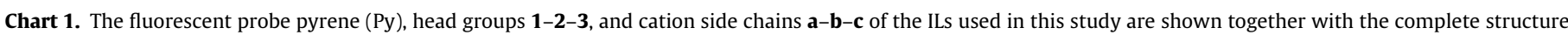
of one representative family $\mathbf{1 a - f}$.

where $I_{0}$ and $I$ denote the fluorescence intensities in the absence and presence of ionic liquid 1a, respectively (see Supplementary Fig. S3).

Imidazolium ILs are weakly fluorescent and their absorption is not significant as compared with Py monomer. Before data treatment ILs fluorescence emission spectra (see Supplementary Fig. S2) were subtracted from Py spectra. The obtained spectra are in agreement with data present in the literature [41]. Fluorescence scattering measurements were recorded with the spectrofluorometer mentioned above, the emission spectra excited at $400 \mathrm{~nm}$ were recorded in the 380-420 $\mathrm{nm}$ range (see Supplementary Fig. S8).
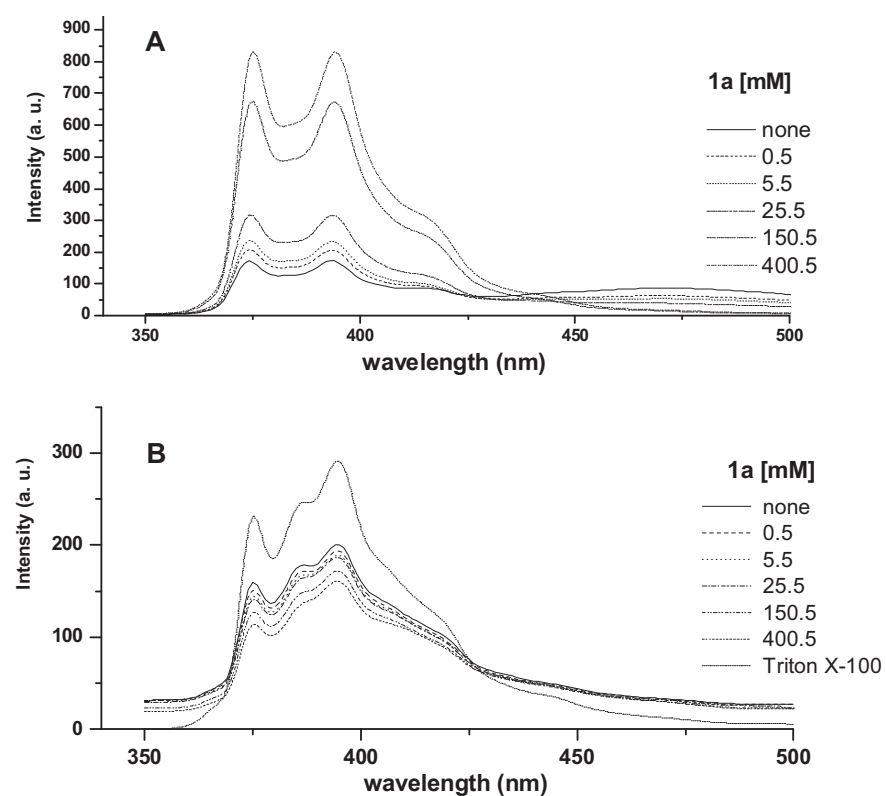

Fig. 1. Panel A: Overlapped fluorescence emission spectra of Py acquired at different 1a concentrations ( $\mathrm{mM}$ ) in buffer solution without liposomes. Panel B: Overlapped fluorescence emission spectra of Py in liposomes recorded at different 1a concentrations (mM), or with Triton X-100.

\subsection{Critical micellar concentration ( $\mathrm{cmc}$ ) measurements}

The cmc of compounds $\mathbf{1 a}, \mathbf{1 b}, \mathbf{1 c}, \mathbf{1 f}, \mathbf{2 c}$ and $\mathbf{3 c}$ has been measured as stated in the literature [42], using the Boltzmann equation (2) and the $\chi^{2}$ (chi-square) coefficient. In Eq. (2) the dependent variable $y$ corresponds to the Py $I_{1} / I_{3}$ ratio value, the independent variable $(x)$ is the total concentration of the tested compound, $A_{1}$ and $A_{2}$ are the upper and lower limits of the sigmoid, respectively, $x_{0}$ is the center of the sigmoid and the corresponding $\mathrm{cmc}$ value.

$\frac{I_{1}}{I_{3}}=A_{2}+\frac{A_{1}-A_{2}}{1+\exp \left(\left(x-x_{0}\right) / d x\right)}$
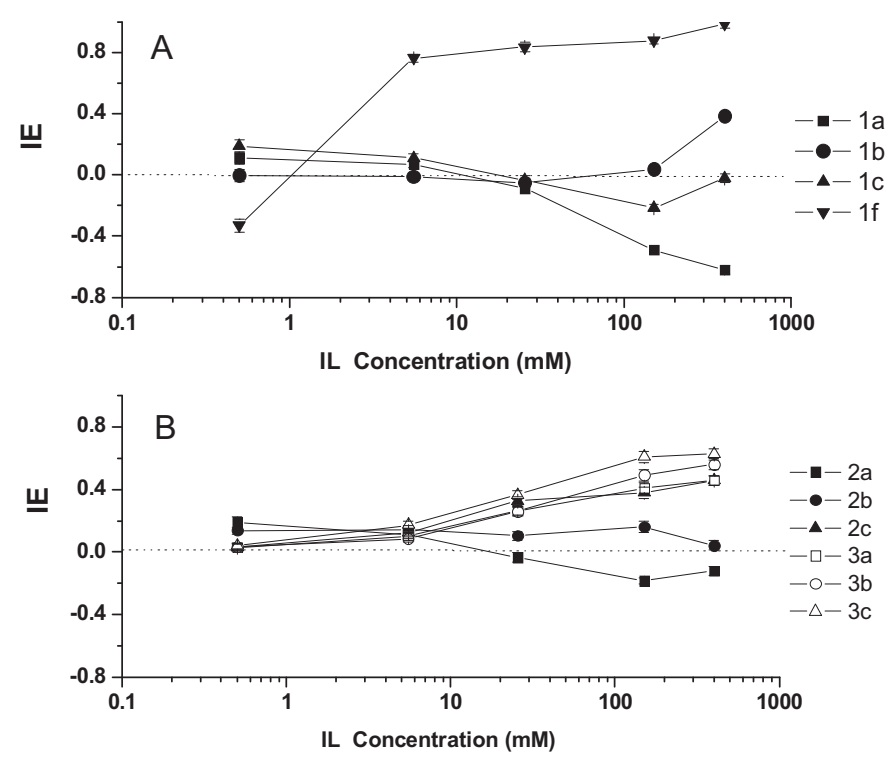

Fig. 2. IE factors. Panel A: N-methylimidazolium chloride ILs 1a-b-c and 1f. Panel B: N-methylpyrrolidinium chloride ILs $\mathbf{2 a - b}-\mathbf{c}$ and 3-methylpyridinium chloride ILs 3a-b-c. Zero lines (dotted line in figure) are depicted as guiding view. 


\subsection{Statistical analysis}

The Primer statistical package (version 6) was used for the statistical evaluation of data. All ILs groups were compared among structure (10 fixed levels, ILs 1a-1c, 2a-2c, 3a-3c, 1f) and concentration (six fixed levels: 0, 0.5, 5.5, 25.5, 150.5, $400.5 \mathrm{mM}$ ). Distance-based two-way permutational analysis of variance PERMANOVA [43] was applied to test for differences related to the investigated factors. Univariate analysis was based on the Euclidean distances on untransformed data. Values were considered significantly different if $p<0.05$. All data are means of at least three replicates and error bars in Figs. 4-7 correspond to standard error values.

\section{Results and discussion}

In a liposomal environment, Py forms excited state dimers (excimers) in a diffusion-limited reaction which depends upon membrane composition, temperature and Py concentration [44]. Furthermore, Py molecules are capable of rapid lateral diffusion, from which the formation of excimers depends. It has been recently reported that Py is predominantly localized in the interfacial region of the membrane [45].

Typical features of the emission spectra of Py are the maxima at 375,385 , and $395 \mathrm{~nm}$ due to vibronic bands, known to be environmentally sensitive [46] and this property has been effectively used for evaluating the polarity of microenvironments in which Py is dissolved [47]. The ratio of the first $(375 \mathrm{~nm})$ and third $(385 \mathrm{~nm})$

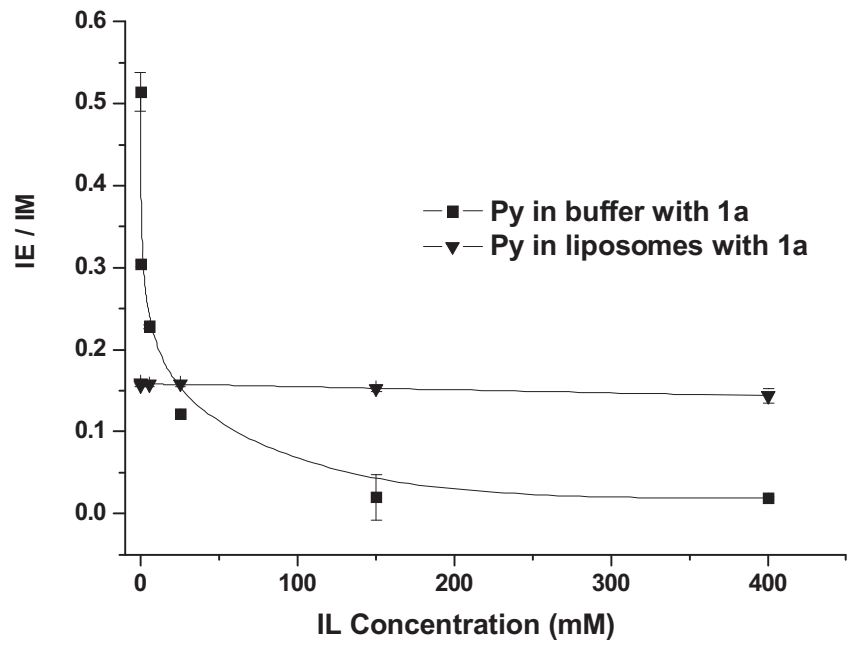

Fig. 3. IE/IM ratio of Py in buffer ( $\boldsymbol{\square})$ and in liposomes ( $\boldsymbol{\nabla})$ in presence of IL 1a.

vibronic peak intensities $\left(I_{1} / I_{3}\right)$ in the Py emission spectrum provides a measure of the apparent polarity of the environment: an increase in the $I_{1} / I_{3}$ ratio is indicative of an increased polarity, while a decrease is indicative of a reduction of polarity, for example the presence of an organized lipidic environment. It is fundamental to point out that the $I_{1} / I_{3}$ ratio considerably varies in different experimental settings depending on the optical configuration used for the
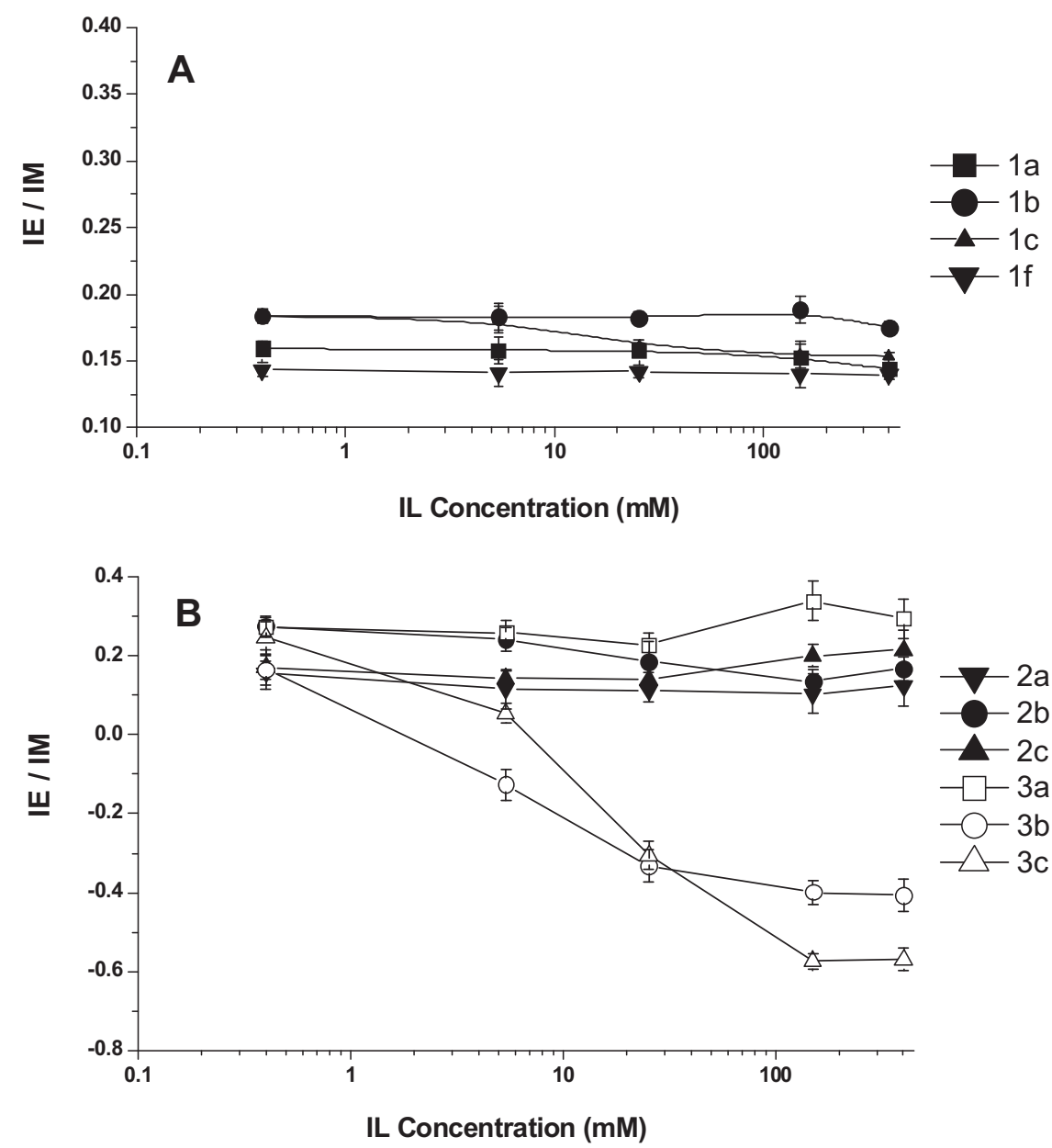

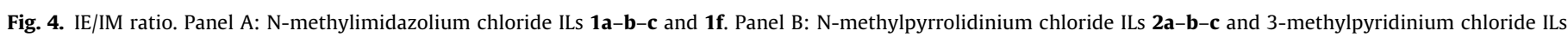
3a-b-c. 
measurements (front-face or right-angle geometry and slits bandwidth), Py concentration, temperature and ionic strength of the solution [48].

Fig. 1A shows the fluorescence emission spectra of Py in buffer solution (spectra of Py at different concentrations, $3 \mu \mathrm{M}, 1.5 \mu \mathrm{M}$, $0.75 \mu \mathrm{M}$ and in different solvents are reported for comparison in the Supplementary Figs. S3 and S4) at different concentrations of 1a ranging from $0.5 \mathrm{mM}$ to $400.5 \mathrm{mM}$ in absence of egg-PC liposomes. Without egg-PC liposomes high concentrations of ILs (150.5 and $400.5 \mathrm{mM}$ ) enhance the emission spectrum of Py, creating a decrease in the polarity of the environment where Py is solubilized, as indicated by the $I_{1} / I_{3}$ ratio value (around 1.3 ) depicted in Supplementary Fig. S6. The $I_{1} / I_{3}$ ratio value is in line with the expected ratio for buffer solutions [49] while in Fig. 1A is evident the effect of $\mathbf{1 a}$ on the formation of excimer population with increasing concentrations of the IL. The effect is particularly evident at high concentration of 1a, indicating a decrease in Py mobility. The excimer emission peak - generally present in the $460-500 \mathrm{~nm}$ emission region - decreases in intensity by increasing the IL concentration. The mobility of the probe diminishes with increasing 1a concentration, behavior confirmed by the IE/IM trend visible in Fig. 3. A similar behavior was observed for the strictly related [BMIM] $\left[\mathrm{PF}_{6}\right][45]$.

To test if the decrease in Py emission spectra can be attributed to quenching phenomena we have exposed Py containing buffer solutions at increasing concentrations of $\mathbf{1 a}$; data are reported in Supplementary Fig. S3. It is worth noting that excimer emission peak is clearly visible in Py emission spectra only when the fluorophore is present at a concentration of $3 \mu \mathrm{M}$. Furthermore, we have compared the $\Delta I$ values against 1a concentration in buffer solution and in egg-PC liposomes (where $\Delta I=I-I_{0}: I_{0}$ and $I$ denote the fluorescence intensities in the absence and presence of ionic liquid 1a, respectively). Considering data in Supplementary Fig. S3, we can affirm that the decrease in Py fluorescence intensity only corresponds to its environment and mobility, we exclude the possibility that quenching of Py occurs. Py forms excimers with very specific fluorescence characteristics, and the ratio of excimer to monomer is known to be dependent on membrane dynamics [50,51]. Fig. 1B shows the behavior of Py emission spectrum in eggPC liposomes exposed at different concentrations of 1a together with the spectrum obtained at $0.1 \%$ concentration of Triton X100.

In Fig. 1B it is possible to observe the increase in the intensity of the peak due to monomer emission (IM) and the decrease of the excimer emission (IE) after the injection of Triton X-100, a surface active substance chosen as reference compound. Excimer emission can be used to calculate the IE factor.

\subsection{IE factor}

The IE factor represents the extent of variation in excimer intensity compared with the excimer intensity obtained after the exposure to Triton X-100 [39]. Negative values of IE highlight higher extent of excimer formation, while positive values denote a lower extent of excimer formation. ILs effects on membrane fusion, expressed as IE factor, are reported in Fig. 2. IE factor has a value of zero when there are not differences in terms of membrane fusion in comparison with untreated samples (dotted line in Fig. 2).

ILs are grouped according to the cationic head structure: imidazolium (Fig. 2A), pyrrolidinium and pyridinium (Fig. 2B). Each group contains ILs with a different composition in the alkyl side chain of
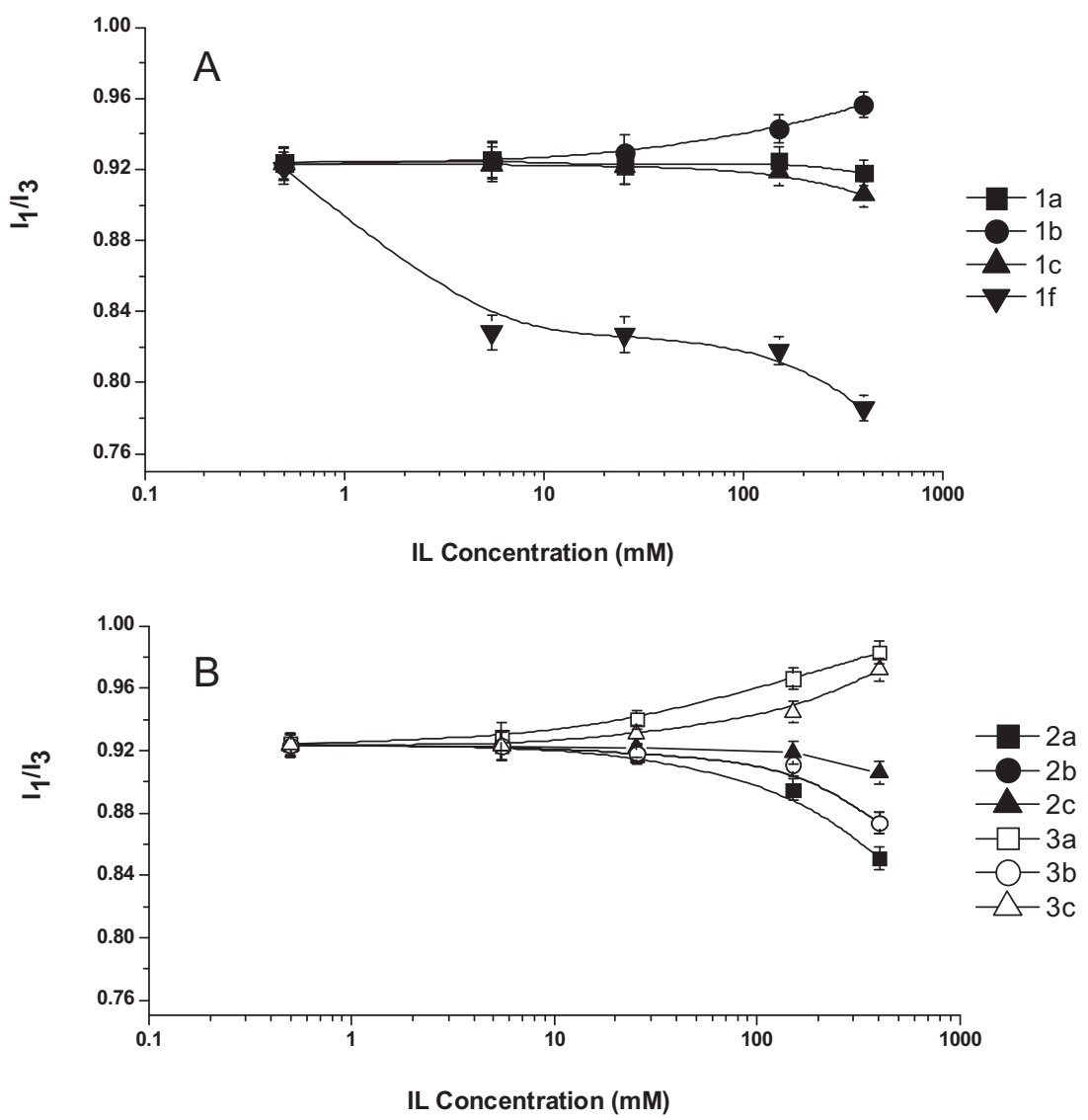

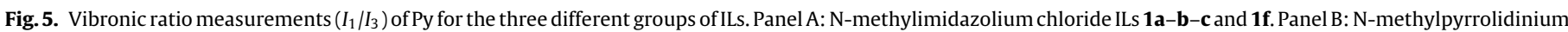
chloride ILs 2a-b-c and 3-methylpyridinium chloride ILs 3a-b-c. 

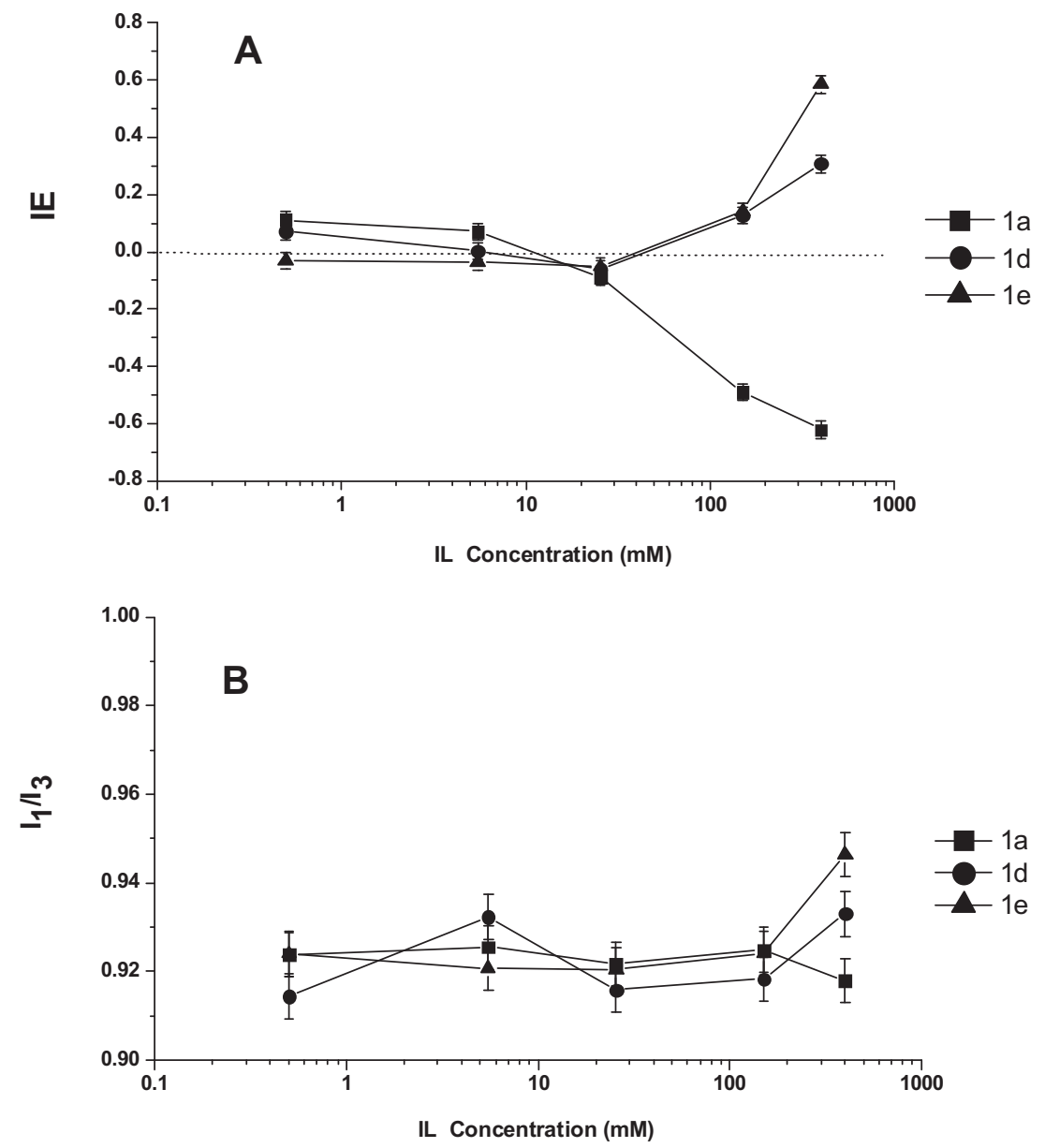

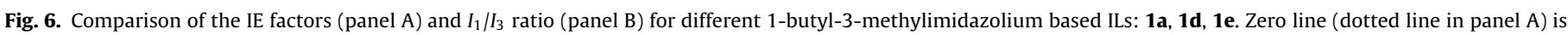
depicted as guiding view.

the cation: without oxygen atoms (1a, $\mathbf{2 a}, \mathbf{3 a})$, with one (1) $\mathbf{1} \mathbf{2} \mathbf{b}, \mathbf{3 b})$ or two ethoxy (1c, 2c, 3c) functionalities in the side chain. The effect obtained through the highly amphiphilic $\mathbf{1 f}$ is added for comparison in Fig. 2A.

As can be seen in Fig. 2A, $1 \mathbf{f}$ produces a decrease in the IE ratio in the concentration range from $0.5 \mathrm{mM}$ to $1 \mathrm{mM}$, suggesting an alteration of the double layer without disruption of the egg-PC liposome and an increase in pyrene lateral mobility (diffusion coefficient) due to a change in lipid packing. We attribute this behavior to the insertion of the long side chains of the ILs cation in the double layer of the egg-PC liposomes that facilitates the formation of excimer populations. At concentrations higher than $1 \mathrm{mM}$ the long alkyl chain of $\mathbf{1 f}$ creates a strong destabilization of the egg-PC liposome, followed by the release of Py monomers and the disruption of the lipid aggregates; in the meanwhile $\mathbf{1 f}$ creates micelles that incorporate Py molecules.

All other tested ILs (1a-1c, 2a-2c, 3a-3c) have an opposite behavior; the insertion of short alkyl chains or ethoxylated chains in the egg-PC liposome wall decreases the excimer population at low ILs concentration (increase in IE factor). At concentrations higher than $25 \mathrm{mM}$ for imidazolium ILs (Fig. 2A) and $5 \mathrm{mM}$ for pyrrolidinium ILs (Fig. 2B) it is not possible to identify a simple trend. Compounds $\mathbf{2 b}, \mathbf{2 c}, \mathbf{3 a}, \mathbf{3 b}$ and $\mathbf{3 c}$ decrease the excimer populations in egg-PC liposomes in the tested concentration interval. We suggest that the interaction of ILs cations with the lipidic double layers creates events that locally produce increased probe concentrations, which enhances the probability of excimer formation and the observed IE ratio, but could alter local diffusion rates in ways that increase or decrease the IE ratio.
An uniform trend of decrease in IE populations (increase in IE factor) can be identified only for pyridinium ILs 3a-3c (Fig. 2B), for which the decrease in excimer population raises the $60 \%$ when compared to Triton X-100 and increases with the increase of the length of the cation side chain. Statistical analysis highlights that the differences in the behavior of IE values for the ILs 1a-1c, $\mathbf{2 a - 2 c}$, $\mathbf{3 a}-\mathbf{3 c}$ are highly significant among ILs in each group and at each concentration.

\subsection{IE/IM ratio}

Another commonly used parameter is the ratio between the excimer and the monomer fluorescence intensity (IE/IM ratio) and it is an indicative parameter of lateral diffusion of Py monomer into lipids bilayers [50,51]. The greater is the extent of excimer formation, the higher is the fluidity and the mobility of lipids molecules in the layers of the membrane.

The trends of IE/IM ratio for Py exposed to different concentration of ionic liquid 1a in buffer or in egg-PC liposomes are visible in Fig. 3. It can be seen that the behavior of Py in buffer (squares) is very sensitive to the presence of IL 1a, while the addition of progressive amounts of IL 1a in egg-PC liposome (triangles) does not substantially change the dynamics of Py aggregates. In buffer solution the lateral mobility of Py is strongly suppressed, and the decrease of excimer population is related to the polarity of the medium and to the increasing concentration of the IL (IE/IM value drops from 0.5 in the absence of $1 \mathbf{a}$ below 0.1 at the presence of the higher concentration tested). This trend correlates with the membrane behavior of IL 1a, which does not deeply alter the double layer 

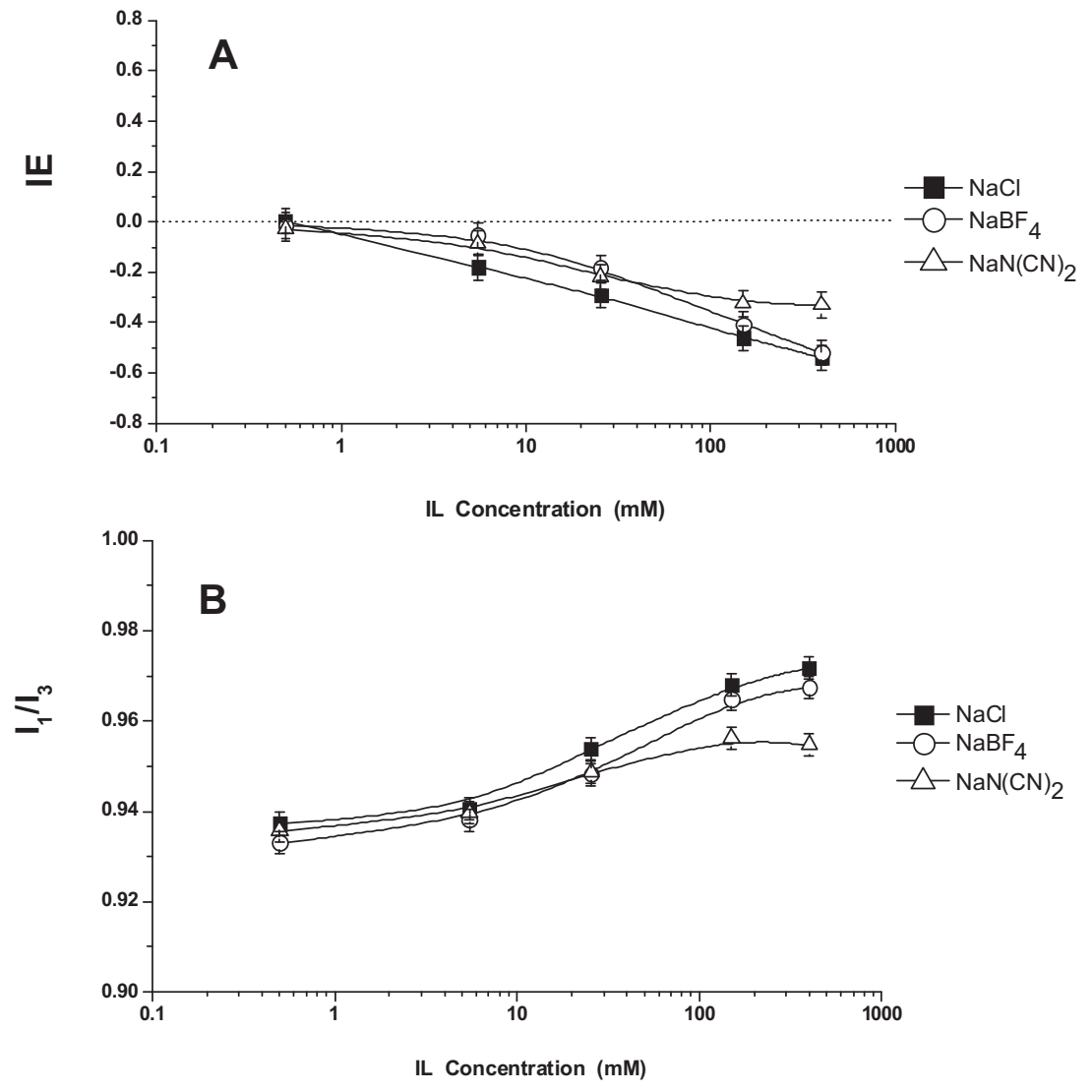

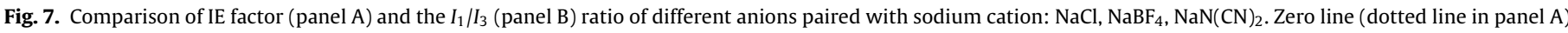
is depicted as guiding view.

packing and membrane integrity even at high concentrations (see Fig. 2A).

The IE/IM ratios against concentration for the three families of ILs are reported in Fig. 4. The effect of different structural motifs of ILs is evident in the different trends. Independently by the cationic head group, ILs generate fewer excimer populations when bearing mono and dioxygenated chains (with exception of 3c). Changes of lower intensities are recorded for ILs with one oxygen atom in the lateral side chain of the cation ( $\mathbf{1 b}$ and $\mathbf{3 b}$ ). Pyrrolidinium ILs have a big difference between the behavior of short alkyl chains IL 2a and ILs with one or two oxygen atoms $\mathbf{2 b}$ and $\mathbf{3 b}$. It is interesting to notice the behavior of IE/IM ratio of the compound 1f. In Fig. $4 \mathrm{~A}$ it is visible that the behavior is not different from substance 1a, suggesting that there is no change in the ratio and mobility of the monomer and excimer populations.

\section{3. $I_{1} / I_{3}$ ratio}

The relative intensity of vibronic bands $I_{1} / I_{3}$ of Py fluorescence in egg-PC liposomes solutions are plotted in Fig. 5. In this case Py was used as a molecular probe to estimate alterations of local micropolarity of the egg-PC membranes caused by incorporation of ILs. It is possible to notice that the ratio values here recorded in egg-PC liposomes, around 0.92 , are in good agreement with those observed for similar lipidic systems and instrumental slits bandpass (0.93-0.96) [40]. The ratio $I_{1} / I_{3}$ is expected to rapidly increase, when the environment of Py changes from highly hydrophobic composed by lipid acyl chains of original egg-PC liposomes, to more hydrophilic (formed in the presence of a polar modifier) [48]. However, in the presence of micelles and other aggregate systems, Py is preferentially solubilized in the interior hydrophobic regions of these aggregates because of poor Py's solubility in water and ratio $I_{1} / I_{3}$ is expected to decrease.

The observed trend for 1f clearly shows the formation of micelles: cmc is reached at a concentration of about $1.7 \mathrm{mM}$ in the presence of liposomes when the $I_{1} / I_{3}$ ratio value rapidly decreases from 0.92 to 0.78 . In buffer solution the cmc of 1 f is $1.7 \mathrm{mM}$ (see Supplementary Fig. S5) and is in line with cmc data obtained for similar buffer solutions ( $\mathrm{NaCl} 0.5 \mathrm{M}$ ) presented in the literature $(1.4 \mathrm{mM})$ [31]. Cmc value in presence of liposomes is lower than data referred to pure water solutions [29,31]; this observation is consistent with the lowering of the $\mathrm{cmc}$ in solutions having high ionic strength. Indeed, salts can lower repulsions between lipid polar heads and facilitate micelle formation. From the trends observed in Fig. 5 we can exclude that critical micellar concentration is reached for all other ILs with the exception of $\mathbf{1 f}$.

A small increase in $I_{1} / I_{3}$ ratio is observable for the ILs $\mathbf{1 b}, \mathbf{3 a}$ and 3c, suggesting that these compounds are able to modify the egg-PC liposome double layer, causing a small increase in the polarity of Py's microenvironment. This increase is visible only for ILs concentrations higher than $50 \mathrm{mM}$. To verify this trend we have measured the $I_{1} / I_{3}$ ratio for compounds $\mathbf{1 a}, \mathbf{1 b}, \mathbf{1 c}, \mathbf{2 c}$ and $\mathbf{3 c}$ in buffer solution (Supplementary Fig. S7) in the $0-450 \mathrm{mM}$ concentration interval. Compounds $\mathbf{1 a}$ and $\mathbf{1 b}$ do not considerably alter the $I_{1} / I_{3}$ ratio in the tested concentration interval and we can confirm that the $\mathrm{cmc}$ is not reached till a concentration of $900 \mathrm{mM}$ [52], both in buffer solution and in water (see Supplementary Fig. S6).

ILs with dioxygenated side chains (1c, $2 \mathbf{c}$ and $\mathbf{3 c}$ ) produce an effect on the $I_{1} / I_{3}$ ratio but do not reach the $\mathrm{cmc}$ at the tested concentrations in buffer solution and the effect is related with the nature of the cationic head group. In the group of dioxygenated ILs, compound 3c, which bears a pyridinium head group, determines 


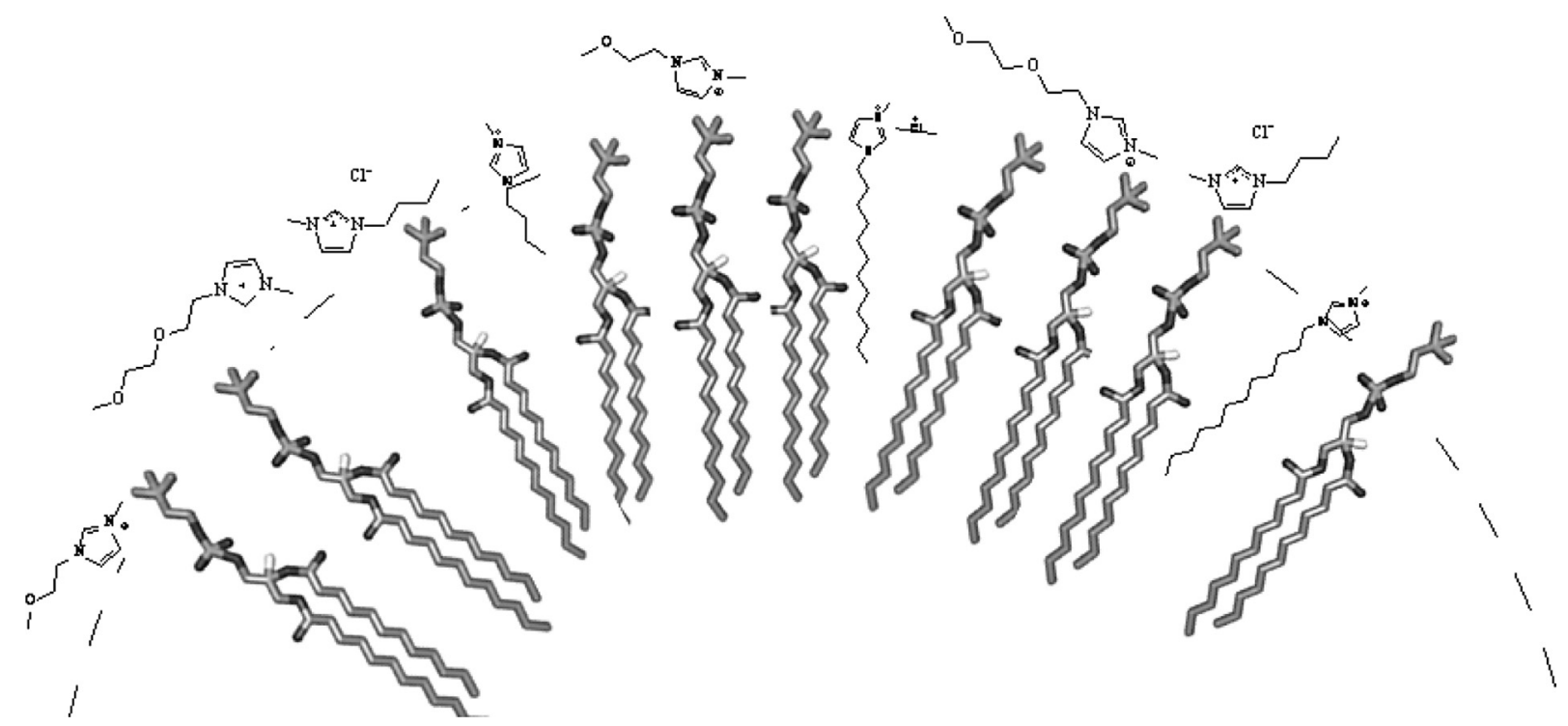

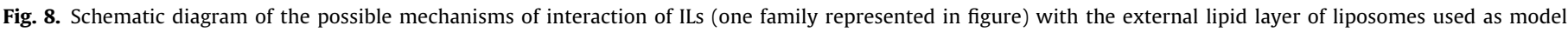
membrane.

the more pronounced effect on $I_{1} / I_{3}$ ratio, creating a decrease from a value of 1.38 to a value 1.16 .

Egg-PC liposomes fluorescence scattering measurements, in the presence of different ILs at increasing concentrations are in agreement with the findings shown above and do not present relevant changes with the expected exception of $\mathbf{1 f}$ (Supplementary Fig. S8), where the scattering value abruptly decreases in correspondence of the $\mathrm{cmc}$, confirming the precedent value of $1.7 \mathrm{mM}$, because of the disruption of the egg-PC liposomes.

\subsection{Anion effect}

Pairing of typical cationic moieties with different anions has been widely used as a strategy for obtaining ionic liquids with similar structure but different physico-chemical properties, like water solubility, lipophilicity and melting point. To assess ILs anion effect on the aggregation properties of egg-PC liposomes, we exposed Py loaded egg-PC liposomes to different concentrations of 1-butyl3-methylimidazolium ILs paired with different common anions (Fig. 6) and to the corresponding sodium salts of the same anions (Fig. 7).

The effects on IE factor and $I_{1} / I_{3}$ ratio of different anions were investigated by checking three 1-butyl-3-methylimidazolium ILs: the chloride 1a, the tetrafluoroborate 1d and the dicyanamide 1e (Fig. 6). At concentrations lower than $25 \mathrm{mM}$ all ILs have similar effects on both the IE factor and $I_{1} / I_{3}$ ratio, but their behavior becomes very different at higher concentrations: 1d and 1e create a much deeper extent of fusion than 1a. This trend could be related to toxicity data where ILs with chloride anion are always less toxic than the corresponding tetrafluoroborate and dicyanamide ones [5].

In order to assess if the effects here described can be solely attributed to the features of the anion or to its ion-paring with 1butyl-3-methylimidazolium, we also measured the effect of $\mathrm{NaCl}$, $\mathrm{NaBF}_{4}$ and $\mathrm{NaN}(\mathrm{CN})_{2}$ on IE factor and on the $I_{1} / I_{3}$ ratio (Fig. 7 ). In the whole range of observed concentrations, the IE factor is lowered by the addition of each sodium salt with a direct dependence on concentration. The lowering of IE factor and the increase in $I_{1} / I_{3}$ ratio at higher salt concentration are tentatively attributed to an increase of the ionic strength of the solution that pushes lipid aggregation irrespective of the anion type [53]. The observed trends in Fig. 6 for ILs 1a, 1d and 1e clearly indicate that these salts behave as ionic pairs in their interaction with lipidic membranes and this behavior is significantly more important for the large less polar anions $\mathrm{BF}_{4}$ and $\mathrm{N}(\mathrm{CN})_{2}$.

Considering the presented data we suggest to summarize the interactions of the ILs with egg-PC model membranes as depicted in Fig. 8. ILs behavior is mainly dependent on the nature of the alkyl chain and the cationic head group and at a lower extent to the anion nature. ILs with long alkyl chains easily insert their hydrophobic tail into the double layer and deeply alter the packing of the lipids at concentration lower than millimolar. Such behavior is registered for shorter-chain ILs. These last ones, similar in structure to $\mathbf{1 a}$ (or 2a and 3a), interact with the double layer only at much higher concentrations and probably are positioned at the water/lipid interface and into the double layer; at these concentrations, they do not disrupt the lipid layers but can alter their fluidity. ILs with polar long oxygenated side chains probably remain in the water compartment at a higher extent, due to their hydrophilicity and can more deeply interact with the external layer of the lipids, probably through ion coupling with the negatively charged heads of model membranes.

\section{Conclusions}

All tested ILs are able to induce fusion of egg-PC liposomes and alter lipid packing. However, as clearly indicated by the IE factor, the IM/IE ratio and the $I_{1} / I_{3}$ ratio values, the ILs tested cannot disrupt the bilayer at concentration up to $0.5 \mathrm{M}$ with the exception of 1f, which acts as a surfactant and disrupts the egg-PC liposomes determining a great extent of membrane fusion also at low concentration. ILs that bear dioxygenated side chains do not aggregate at the tested concentrations in buffer solution but alter the lipid packing and this phenomenon is in line with IE factor values recorded at high concentrations (150 and $400 \mathrm{mM}$ ). Between the three ILs groups, pyridinium ILs proved to be the most membrane active compounds. At least for imidazolinium ILs, ion paring prevents the instauration of significant ionic strength effects for concentrations below $25 \mathrm{mM}$ and different ILs' anions do not play an important role in determining the membrane activity in the same concentration range.

Due to their multiple possible physiological targets, the mechanism of ILs toxicity remains somewhat obscure, although the interaction of ILs with cellular membranes is still the most important expected effect. In this contribution we bring data that indicate 
the interaction of ILs with lipids, highlighting the type of interaction, and the molecular motifs that are responsible for ILs activity. The comprehension of the specific mechanism of ILs action is a key step for the design of newly structured substances possessing reduced toxicity whose application stands in the field of colloids and soft materials. We believe that this work on the interaction between ILs and egg-PC liposomes can represent the basis for the future use of model phospholipids bilayers in structure-activity relationships studies.

\section{Acknowledgements}

We acknowledge the Ministero dell'Istruzione dell'Università e della Ricerca (MiUR) and the University of Bologna (Campus di Ravenna) for funding. We thank Regione Emilia Romagna (PORFESR grant, Progetto Tecnopoli) for fellowship to D. Malferrari and C. Samorì. We acknowledge Giulia Balestrelli and Prof. Giuseppe Falini for the support in DLS measurements.

\section{Appendix A. Supplementary data}

Supplementary data associated with this article can be found, in the online version, at http://dx.doi.org/10.1016/j.colsurfb. 2014.11.021.

\section{References}

[1] R.D. Rogers, G.A. Voth, Acc. Chem. Res, 40 (2007) 1077.

[2] H. Weingartner, Angew. Chem. Int. Ed. 47 (2008) 654.

[3] J. Hallett, T. Welton, Chem. Rev. 111 (2011) 3508.

[4] N.V. Plechkova, K.R. Seddon, Chem. Soc. Rev. 37 (2008) 123.

[5] J. Ranke, S. Stolte, R. Stormann, J. Arning, B. Jastorff, Chem. Rev. 107 (2007) 2183.

[6] T.P. Pham, C.W. Cho, Y.S. Yun, Water Res. 44 (2010) 352.

[7] K.M. Docherty, C.F. Kulpa, Green Chem. 7 (2005) 185.

[8] D. Coleman, N. Gathergood, Chem. Soc. Rev. 39 (2010) 600

[9] P. Stepnowski, S. Stolte, Curr. Org. Chem. 15 (2011) 1871.

[10] P. Stepnowski, P. Storoniak, Environ. Sci. Pollut. 12 (2005) 199

[11] C.W. Cho, U. Preiss, C. Jungnickel, S. Stolte, J. Arning, J. Ranke, A. Klamt, J. Krossing, J. Thoming, J. Phys. Chem. B 115 (2011) 6040.

[12] P. Stepnowski, W. Mrozik, J. Nichthauser, Environ. Sci. Technol. 41 (2007) 511.

[13] P. Stepnowski, Aust. J. Chem. 58 (2005) 170.

[14] H. Sifaoui, K. Lugowska, U. Domańska, A. Modaressi, M. Rogalski, J. Colloid Interface Sci. 314 (2007) 643.

[15] K.P. Kootery, H. Jiang, S. Kolusheva, T.P. Vinod, M. Ritenberg, L. Zeiri, R. Volinsky, D. Malferrari, P. Galletti, E. Tagliavini, R. Jelinek, ACS Appl. Mater. Interfaces 6 (2014) 8613

[16] R.T. Derea, R.R. Pala, S.P. Patila, M. Salunkhe, Tetrahedron Lett. 44 (2003) 5351.
[17] T.L. Greaves, C.J. Drummond, Chem. Soc. Rev. 37 (2008) 1709.

[18] J.L. Anderson, V. Pino, E.C. Hagberg, V.V. Shares, D.W. Armstrong, Chem. Commun. 19 (2003) 2444.

[19] Y. Gao, N. Li, L. Zheng, X. Bai, L. Yu, X. Zhao, J. Zhang, M. Zhao, Z. Li, J. Phys. Chem. B 111 (2007) 2506

[20] Z. Miskolczy, K. Sebok-Nagy, L. Biczok, S. Gokturk, Chem. Phys. Lett. 400 (2004) 296.

[21] C. Samorì, D. Malferrari, P. Valbonesi, A. Montecavalli, F. Moretti, P. Galletti, G Sartor, E. Tagliavini, E. Fabbri, A. Pasteris, Ecotoxicol. Environ. Saf. 73 (2010) 1456.

[22] K.O. Evans, Colloids Surf. A: Physicochem. Eng. Asp. 274 (2006) 11.

[23] S. Jeong, S. Ho Ha, S.-H. Han, M.-C. Lim, S.M. Kim, Y.-R. Kim, Y.-M. Koo, J.-S. So, T.-J. Jeon, Soft Matter 8 (2012) 5501.

[24] D. Patra, E. El Khoury, D. Ahmadieh, S. Darwish, R.M. Tafech, Photochem. Photobiol. 88 (2012) 317.

[25] E.D. El Khoury, D. Patra, J. Phys. Chem. B 117 (2013) 9699

[26] N. Gal, D. Malferrari, S. Kolusheva, P. Galletti, E. Tagliavini, R. Jelinek, Biochim. Biophys. Acta 12 (2012) 2967

[27] G. Cevc, H. Richardsen, Adv. Drug. Deliver. Rev. 38 (1999) 207

[28] G. Sessa, G. Weissmann, J. Lipid Res. 9 (1968) 310.

[29] M. Blesic, M.H. Marquez, N.V. Plechkova, K.R. Seddon, L.P.N. Rebelo, A. Lopes, Green Chem. 9 (2007) 481.

[30] J. Wang, H. Wang, S. Zhang, H. Zhang, J. Zhao, J. Phys. Chem. B 111 (2007) 6181

[31] B. Dong, N. Li, L.Q. Zheng, L. Yu, T. Inoue, Langmuir 23 (2007) 4178.

[32] A. Pinkert, K.N. Marsh, S. Pang, M.P. Staiger, Chem. Rev. 109 (2009) 6712.

[33] P. Galletti, F. Moretti, C. Samorì, E. Tagliavini, Green Chem. 9 (2007) 987.

[34] L. Branco, C.A.M. Afonso, J. Org. Chem. 69 (2004) 4381.

[35] E. Stathatos, P. Lianos, V. Jovanovski, B. Orel, J. Photochem. Photobiol. A 169 (2005) 57.

[36] H.S. Schrekker, M.A. Gelesky, M.P. Stracke, C.M.L. Schrekker, G. Machado, S.R. Teixeira, J.C. Rubim, D.J. Jairton, J. Colloid Interface Sci. 316 (2007) 189.

[37] C. Samorì, A. Pasteris, P. Galletti, E. Tagliavini, Environ. Toxicol. Chem. 26 (2007) 2379.

[38] A. Modelli, A. Sali, P. Galletti, C. Samorì, Chemosphere 73 (2008) 1322.

[39] P. Schoen, L. Leserman, J. Wilschut, FEBS Lett. 390 (1996) 315.

[40] S. Shrivastava, Y.D. Paila, A. Dutta, A. Chattopadhyay, Biochemistry 47 (2008) 5668.

[41] P.K. Mandal, A. Paul, A. Samanta, J. Photochem. Photobiol. A 182 (2006) 113.

[42] J. Aguiar, P. Carpena, J.A. Molina-Bolivar, C. Carnero Ruiz, J. Colloid Interface Sci. 258 (2003) 116.

[43] M.J. Anderson, C.J.F. ter Braak, J. Stat. Comput. Simul. 73 (2003) 85.

[44] F.M. Winnik, Chem. Rev. 93 (1993) 587.

[45] M. Herrenbauer, Biosorption of Polycyclic Aromatic Hydrocarbons (PAH) to Microorganisms and Liposomes, Shaker Verlag, Aachen, Germany, 2002.

[46] K. Kalyanasundaram, J.K. Thomas, J. Am. Chem. Soc. 99 (1977) 2039

[47] Y. Waka, K. Hamamoto, N. Mataga, Photochem. Photobiol. 32 (1980) 27.

[48] S. Pandey, S.N. Baker, S. Pandey, G.A. Baker, J. Fluoresc. 22 (2012) 1343.

[49] N.I. Zahid, O.K. Abou-Zied, R. Hashim, T. Heidelberg, J. Phys. Chem. C 115 (2011) 19805.

[50] J.M. Vanderkooi, J.B. Callis, Biochemistry 13 (1974) 4000.

[51] V. Ioffe, G.P. Gorbenko, Biophys. Chem. 114 (2005) 199.

[52] C. Jungnickel, J. Luczak, J. Ranke, J.F. Fernandez, A. Muller, J. Thoming, Colloids Surf. A 316 (2008) 278

[53] M. Blesic, A. Lopes, E. Melo, Z. Petrovski, N.V. Plechkova, J.N. Canongia Lopes, K.R. Seddon, L.P.N. Rebelo, J. Phys. Chem. B 112 (2008) 8645. 